\title{
Acumuladores de animais: instrumento de vistoria técnica e perfil de casos no município de Guarulhos, SP, Brasil*
}

\section{Animal hoarders: Technical inspection instrument and profile in the city of Guarulhos, SP, Brazil}

\author{
Tadeu Campioni Morone Cardoso, ${ }^{* *}$ Paula Andrea de Santis Bastos***
}

\begin{abstract}
Resumo
O transtorno de acumulação de animais, é uma psicopatologia caracterizada pela aquisição de muitos animais mantidos em espaços inadequados, onde os cuidados básicos de saúde, alimentação e bem-estar são negligenciados. O objetivo deste estudo foi caracterizar o perfil de acumuladores de animais no município de Guarulhos e elaborar um instrumento de coleta de dados para ser utilizado em vistoria técnica nestes casos. Foi realizado um levantamento e análise descritiva dos arquivos de casos de acumuladores de animais atendidos pelo serviço municipal. Dentre os 26 casos recebidos no período de 2012-2017, $12(46,16 \%)$ eram referentes a casos de acumulação de animais. Estas ocorrências foram encaminhadas ao serviço de saúde como denúncias. Os dados obtidos, por meio das análises dos prontuários, foram refinados e, posteriormente, transcritos em planilhas considerando as variáveis de interesse, assim, facilitando o processo de interpretação. Para caracterizar o perfil dos casos de acumuladores, os dados adquiridos foram analisados por meio de cálculos de médias e frequências relativas. O perfil de acumuladores se caracterizou por mulheres, solteiras, idosas, desempregadas ou aposentadas, que residem sozinha em casa própria, portadoras de comorbidades ou fatores psicológicos associados, acumulando cães e gatos, especificamente, cães em sua maioria. O instrumento de vistoria em atenção a indivíduos em situação de acúmulo de animais proposto apresenta caracterização do indivíduo, ambiente, condições gerais dos animais e, ainda, indicadores de comprometimento de bem-estar animal ou maus-tratos.
\end{abstract}

Palavras-chave: Saúde única. Saúde pública. Transtorno de acumulação.

\begin{abstract}
Animal hoarding disorder is a psychopathy characterized by the acquisition of many animals that are kept in improper locations, in which the basic health care, feeding and well-being are neglected. The objective of this study was to characterize the profile of animal hoarders in the city of Guarulhos and to elaborate an instrument of data collection to be used in technical survey in cases of animal hoarding. Amongst the 26 cases analyzed, 12 (46,16\%) were related to animal hoarding. These cases were sent to the health care service as denounces. Data obtained through analysis of medical records were refined and later transcribed into spreadsheets considering the variables of interest, thus facilitating the interpretation process. To characterize the profile of the hoarding cases, data were analyzed by means of calculations of means and relative frequencies. Hoarders profile is characterized as female, single, elderly, unemployed or retired, living alone at her own house, with associated comorbidities or psychological disorders, hoarding dogs and cats, specifically dogs in most cases. The proposed instrument presents characterization of the individual, environment, general conditions of the animals and also indicators of impairment of animal welfare or animal abuse.

Keywords: One Health. Public Heatlh. Hoarding disorder.

\section{Introdução}

O acúmulo de animais é considerado um transtorno existente em todas as sociedades (Lima, 2011). Acredita-se que ele esteja presente em cerca de $2 \%$ a $6 \%$ da população mundial (APA, 2014), porém, de acordo com levantamento realizado nos principais bancos de dados científicos (Capes, Elsevier, PubMed, Medline, Google Acadêmico e Banco de teses e

dissertações USP e UNESP), pode-se verificar a escassez de trabalhos significativos sobre o cenário nacional referente a esta temática. $\mathrm{O}$ conhecimento deste transtorno para correta intervenção é de extrema relevância em nosso país, pois, de acordo com o último levantamento nacional, o Brasil ocupa o segundo lugar no ranking de países com maior população de animais de estimação, totalizando 132 milhões, atrás, apenas, dos Estados Unidos (IBGE, 2013). Outro fator que também deve
\end{abstract}

*Recebido em 18 de janeiro de 2019 e aceito em 16 de setembro de 2019.

**Médico veterinário, mestre em Saúde e Bem-Estar Animal, Programa de Mestrado Profissional em Saúde e Bem-Estar Animal, Complexo Educacional FMU, São Paulo, SP, Brasil. tadeuvet@yahoo.com.br.

***Médica Veterinária, doutora em medicina veterinária, coordenadora do programa de Mestrado Profissional em Saúde e Bem-Estar Animal, Complexo Educacional FMU, São Paulo, SP, Brasil. 
ser levado em consideração é a "posse responsável" de cães e gatos que muitas vezes é negligenciada (Domingues et al, 2013), possivelmente contribuindo para o abandono de animais.

Este transtorno, anteriormente visto como variação do Transtorno Obsessivo Compulsivo (TOC), foi recentemente incluído (2013) no Manual Diagnóstico e Estatístico de Transtornos Mentais (DSM-5) da American Psychiatric Association, caracterizado pela necessidade de coletar e acumular intencionalmente objetos ou animais, promovendo, consequências no ambiente(APA, 2013a), ausência de padrões mínimos de higiene, falta de percepção sobre o problema e negação (Patronek ; Nathansan, 2006, 2009). Os indivíduos portadores deste transtorno, chamados de acumuladores, apresentam dificuldade patológica em se desfazer das posses, mesmo que estas não lhes sejam úteis (Pertusa et al, 2010; Lima, 2011; Mataix-Cols ; Pertusa, 2012; APA2013a; APA, 2013b), apresentando forte tendência em acumular itens aleatórios, acreditando que futuramente poderão ter alguma utilidade, ou devido a algum valor (financeiro ou afetivo), sentemse mais seguros em mantê-los guardados (Patronek ; Nathansan, 2006; APA, 2013a; APA, 2013b; HARC, 2016).

Porém, as condições de vida que eles oferecem a seus animais e o ambiente em que vivem podem ser caracterizadas como maus tratos (Tolin et al, 2008; HARC, 2013). Na maior parte das vezes, os animais, na condição de acúmulo, sofrem de desnutrição por não disporem de alimentação adequada, ou até mesmo pela escassez de alimento, de infecções virais, bacterianas e fúngicas; de parasitoses; diarreia; afecções respiratórias e desidratação. Este cenário e a quantidade de animais em ambiente restrito promovem e disseminam agentes de doenças entre os animais, e, possivelmente, até zoonoses que podem ser transmitidas ao acumulador, vizinhos próximos e aos agentes de saúde que vistoriam o local (Patronek; Nathansan, 2006; Tolin et al, 2008; Mataix-Cols e Pertusa, 2012). Apesar de ser uma psicopatologia, o acúmulo de animais apresenta diferentes cenários, ou seja, existem indivíduos que acumulam centenas de animais, bem como outros que possuem animais em menor número. Consequentemente, as condições destes animais também podem variar. Existem casos em que os animais estão muito debilitados, com a saúde comprometida, e outros que a situação é menos precária, mas em ambos os casos sempre há comprometimento do bem-estar geral, da sanidade dos animais e do ambiente (Patronek ; Nathansan, 2006; Tolin et al, 2008; Mataix-Cols ; Pertusa, 2012). Frequentemente estes acumuladores acabam se envolvendo em processos judiciais devido aos excessos provenientes da acumulação compulsiva (Pogosian, 2010; Lima, 2011; APA, 2013a; APA, 2013b). Estes fatores podem prejudicar e comprometer a vida social dos indivíduos com TA. Por este motivo, eles acabam se isolando e se sentem incomodados com a presença de outras pessoas em sua residência (Steketee ; Frost, 2003; APA, 2013a; APA, 2013b; Dimauro et al, 2013). Cerca de $75 \%$ destes indivíduos apresentam uma comorbidade associada, como o transtorno de humor e/ou de ansiedade (APA, 2014).

Estudos epidemiológicos, observaram que os indivíduos que acumulam geralmente residem sozinhos e possuem baixa qualidade de vida (Grisham et al, 2016). Acredita-se que este comportamento pode estar presente desde a infância ou adolescência, intensificando seus sintomas na fase adulta(Pertusa et al, 2010; APA, 2013a; APA, 2013b; HARC,
2016; Grisham et al, 2016), com forte tendência em agravarse com o passar do tempo e à medida que mais objetos, ou animais, vão sendo acumulados (Patronek, 1999). Estudos americanos indicaram que o perfil destes indivíduos, de modo geral, é composto por mulheres solteiras, de meia-idade e que residem sozinhas (Patronek e Nathansan, 2009; Pertusa et al, 2010). Os animais mais acumulados são cães e gatos, porém, também há relatos de animais de grande porte e exóticos (Mills, 2013). Acredita-se que eventos traumáticos e problemas de relacionamento podem estar associados ao início do TA (Mills, 201). Pesquisadores brasileiros (Rocha et al, 2015) analisaram denúncias de maus tratos aos animais registradas na prefeitura do município de Curitiba, no ano de 2012. No período estudado foram recebidas 2.162 denúncias, sendo que 81 delas $(3,7 \%)$ tratavam de acúmulo de animais (Rocha et al, 2015). Outro levantamento no mesmo município, junto às Secretarias Municipais de Saúde, Meio Ambiente e Assistência Social no período entre 2013 e 2015 constatou que dentre as 226 denúncias, 113 (50\%) foram confirmadas como acúmulo compulsivo, dos quais 48 (42,5\%) de objetos, $41(36,3 \%)$ de animais e $24(21,2 \%)$ objetos e animais(Cunha et al, 2017). Ressalta-se, segundo o conceito de saúde única, a corresponsabilidade de todos, sociedade civil e entidades governamentais e não governamentais (WHO, 2017), diante de problemática que envolva a vulnerabilidade de seres humanos e animais; nesse sentido, é evidente a necessidade de pesquisas e métodos de atuação sobre o tema. No Brasil, as demandas e denúncias envolvendo este transtorno, são atendidas pelo serviço público municipal estabelecido pelo Sistema Único de Saúde, e encaminhado o Centro de Controle de Zoonoses nos municípios dos quais dispõem deste serviço. Sabemos que o enfrentamento à esta situação é multisetorial, envolvendo diversos serviços de saúde, e que a questão da proteção e bem-estar animal, por exemplo, ainda é algo que está em desenvolvimento no sentido de ainda não dispor de um órgão público exclusivo. Porém, atualmente, algumas cidades já vêm estruturando órgãos específicos vinculados à Secretaria do Meio Ambiente, como a Secretaria Especial de Direitos Animais, em Porto Alegre- RS; a Secretaria Executiva dos Direitos Animais em Recife - PE (Tavolaro ; Cortez, 2016), e o Departamento de Proteção Animal de Guarulhos - SP (Guarulhos, 2018). O Sistema Nacional de Vigilância em Saúde e o Sistema Nacional de Vigilância Sanitária pela Portaria $n^{\circ} 1.378$ do Ministério da Saúde, de nove de julho de 2013, que, posteriormente foi revogado pelo consolidado $n^{\circ} 05$ de 28/09/2017, regulamenta as responsabilidades e define diretrizes para a execução e financiamento das ações de vigilância em saúde pela União, Estados, Distrito Federal e municípios. Em complemento, alguns municípios possuem sua legislação municipal que institui seu código sanitário, quando não há, no caso de municípios de menor porte, os mesmos seguem os Códigos Sanitários Estaduais. Um exemplo disso é a Lei Municipal n¹3.725 que institui o Código Sanitário do Município de São Paulo (São Paulo (SP), 2004); e dois anos após, no município de Guarulhos, também foi estabelecido por meio da Lei Municipal $n^{\circ} 6.144$ (Guarulhos (SP), 2006). Apesar da legislação e diretrizes até então existentes, os casos de acumuladores, em especial de animais, não possuem uma padronização de atendimento, coleta de informações, bem como normativas específicas, enquadrando esses casos como processos judicias e autuações recorrentes referentes, apenas, a situação do ambiente e dos 
animais. Esta visão não compreende o indivíduo acumulador como um portador de uma psicopatologia (Pogosian, 2010; Lima, 2011; APA, 2013a; APA, 2013b). Outra particularidade é que, de modo geral, os serviços de saúde realizados nesses casos envolvem o controle populacional de cães e gatos - por meio de castrações - e vacinação antirrábica; sendo que o destino dos animais para um novo ambiente somente ocorre por meio de medida judicial. Ressalta-se que quando ocorrem parcerias entre o município e entidades do terceiro setor há o encaminhamento dos animais para adoção e lares temporários. Diferentemente da legislação brasileira, no cenário norte americano muitos animais acabam sendo encaminhados à eutanásia (Tolin et al, 2008).

Com a finalidade de auxiliar e elaborar um padrão de atendimento em atenção à indivíduos com este transtorno, por parte dos serviços de saúde, em 28 de dezembro de 2016, no município de São Paulo, foi estabelecido o Decreto $n^{\circ} 57.570$ que institui a Política Municipal de Atenção Integral e Multidisciplinar às Pessoas em Situação de Acumulação (São Paulo (SP), 2016). Em Guarulhos, município vizinho, com o objetivo de compreender melhor este transtorno, auxiliar tecnicamente e intervir de maneira integrativa nestes casos, foi criada a Rede Intersetorial de Apoio à Pessoa com Acumulação (RIAPAC), que é composta por integrantes das secretarias municipais de saúde e serviços públicos da cidade. Entretanto, apesar da regulamentação da RIAPAC por meio da Portaria Municipal n76, de 19 de agosto de 2016, e que foi, posteriormente alterada pela Portaria Municipal n 158/2018-SS (Guarulhos (SP), 2018) não há nenhum estudo científico, nem levantamento realizado no município caracterizando os casos atendidos pela rede. Também não há, estruturado e sendo utilizado pela equipe técnica, instrumento que organize a coleta de dados durante a realização de vistoria de casos de indivíduos em situação de acumulação de animais. Ressalta-se que uma boa coleta de dados é fundamental para o diagnóstico de situação, facilitando, em muito, a elaboração de laudo mais completo (Barbosa, 2008).

Um instrumento de coleta de dados, organizadamente elaborado e compatível com a situação e público alvo, pode garantir: baixo custo; redução no grau de intromissão e tendência ao nivelamento social - por parte de quem os coleta; redução da minimização ou exacerbação de problemas; estabilidade da informação; maior confiabilidade dos dados; eficiência para esclarecer questões complexas no desenvolvimento de projetos e ações, contribuindo, assim, para a otimização da coleta, padronização e processamento das informações. Neste sentido, deve-se considerar que todo instrumento que facilite a coleta e organize as informações fomentará o melhor entendimento dos fatores relacionados, casuística, comorbidades, grau de bemestar dos animais; auxiliando, desta forma, o planejamento de ações de atendimento às necessidades das pessoas e animais envolvidos (Barbosa, 2008).

O objetivo geral deste estudo é caracterizar o perfil de acumuladores de animais no município de Guarulhos. Como objetivo específico, este trabalho visa promover a estruturação de um instrumento de coleta de dados para ser utilizado em vistoria técnica, em casos de acúmulo de animais, por agentes de serviços de saúde.

\section{Material e métodos}

Com o objetivo de entender a dinâmica dos casos atendidos e avaliar o processo de coleta de dados, por parte dos agentes sanitários, e consequentemente viabilizar a elaboração do perfil de acumuladores de animais atendidos e a criação do instrumento de vistoria técnica, realizou-se o levantamento e análise descritiva dos arquivos de casos de acumuladores de animais atendidos pela RIAPAC, havendo caracterização do indivíduo com o transtorno que foi, ou está sendo atendido pela rede intersetorial. Foi ponderado trabalhar com os casos de acumuladores de animais atendidos, no período de 2012 a 2017. Cada caso recebe, por parte do serviço de saúde, um número de atendimento e possui prontuário individualizado para acompanhamento do andamento e para aspectos burocráticos. Os dados constantes nestes processos são basicamente: dados demográficos; sociais do denunciado; econômicos; tipo de acumulação; descrição da residência; tempo de atuação da intervenção e do acompanhamento psicológico e desfecho dos casos em relação à pessoa acumuladora. Em relação aos animais, estavam presentes informações referentes à quantidade, espécie e condições gerais e especificas (escore corporal, presença de ectoparasitas, lesões de pele, sinais clínicos aparentes à inspeção física, entre outros) dos animais no domicílio, assim como, do ambiente. Entretanto, os dados presentes foram colhidos de maneira não uniforme, ou seja, nas anotações referentes à visita de um dia havia categoria de informações não presentes nas anotações de outra visita o que dificultou a composição da situação de cada indivíduo. Para organização dos dados, todos eles foram transcritos em planilha Excel®.

Todos os casos atendidos pela Rede foram lidos individualmente, porém, apenas casos de acúmulo de animais foram separados para o processo de coleta de informações. Os dados obtidos, por meio das análises dos prontuários, foram refinados e, posteriormente, transcritos em planilhas considerando as variáveis de interesse, assim, facilitando o processo de interpretação. Os dados padrão coletados basicamente se referiram à origem da denúncia, localização, dados demográficos, sociais, econômicos, presença de comorbidades, animais acumulados, ambiente e andamento do acompanhamento do caso. Para caracterizar o perfil dos casos de acumuladores atendidos, os dados obtidos foram analisados por meio de cálculos de médias e frequências relativas. A partir da verificação dos dados identificou-se a necessidade da elaboração de instrumento de vistoria técnica. Com relação ao desenvolvimento desse instrumento utilizou-se como base a observação e leitura de todos prontuários dos casos atendidos, identificando informações importantes e relevantes. Considerando este processo e o contato pessoal com agentes de saúde atuantes neste cenário pôde-se verificar que dados, tais como: condições do ambiente (sanidade, dificuldade de acesso, presença ou não de fezes, lixo, animais mortos, objetos acumulados e odor) e dos animais (quantidade, escore corporal, ocorrência de diarreia, doenças associadas, presença de lesões de pele, acesso à alimento e água) - que são extremamente relevantes ao acompanhamento do caso, muitas vezes não eram coletadas em uma visita; pois como não há documento orientador na coleta de dados, dependiam da individual interpretação de cada agente, e não de um roteiro a ser seguido. Desta forma, o instrumento elaborado teve como objetivo facilitar a coleta dos dados relevantes, etapa essa, fundamental, para o entendimento e acompanhamento dos casos.

Localizado na região metropolitana de São Paulo, Guarulhos é o segundo maior município paulista. Seu território é dividido 
em 4 regiões de saúde: 1) Centro; 2) Cantareira; 3) São João Bonsucesso; 4) Pimentas-Cumbica (IBGE, 2018). Os dados da RIAPAC ficam armazenados em arquivo do Centro de Controle de Zoonoses do município, que é localizado na região "3".

O pré-projeto foi submetido e aprovado pela Comissão de Avaliação e Acompanhamento de Projetos e Pesquisas da Secretaria da Saúde de Guarulhos, e eletronicamente por meio da Plataforma Brasil, o qual foi aprovado segundo Termo Consubstanciado número 2.297.150. Ressalta-se que, neste trabalho, a identidade e o endereço dos indivíduos atendidos serão sempre mantidos em sigilo. Salienta-se que este estudo se trata de análise descritiva de dados em arquivos, sem a realização de visitas e contato direto com os munícipes ou animais.

\section{Resultados}

Dentre os 26 casos atendidos pela RIAPAC no período exposto, $12(46,16 \%)$ eram referentes à casos de acumulação de animais. Estas ocorrências foram encaminhadas ao serviço de saúde como denúncias. A metade delas, ou seja, 50\% (6/12) vieram por meio do serviço de ouvidoria, $25 \%(3 / 12)$ pela internet, $16,6 \%(2 / 12)$ em memorandos e $8,4 \%(1 / 12)$ por demanda realizada pessoalmente. Os motivos de denúncia registrada foram: excesso de animais $(41,6 \%)$, maus-tratos $(41,6 \%)$, presença de animais sinantrópicos $(8,4 \%)$ e excesso de lixo $(8,4 \%)$. Destes casos, o início da demanda e acompanhamento surgiu conforme ilustra figura 1 :

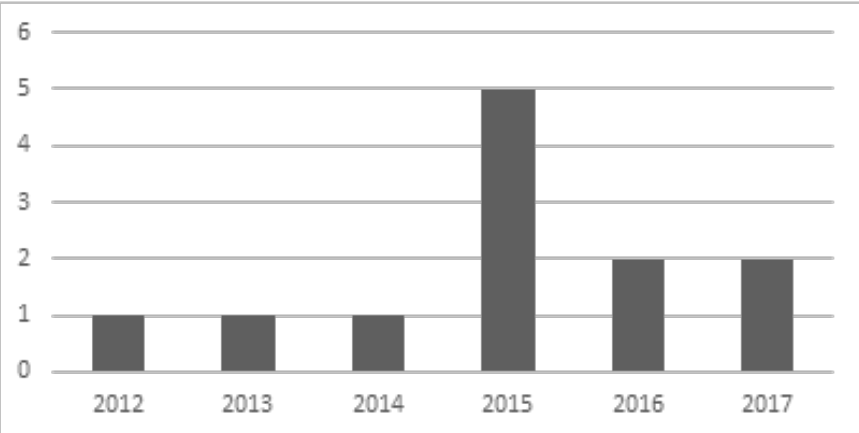

Figura 1: Número de casos de acumuladores de animais atendidos pela RIAPAC por ano

Com relação à localização desses casos no município, a maioria deles $41,7 \%(5 / 12)$ situavam-se na região 3 . A maioria destes, $75 \%(9 / 12)$, situados em casas na área urbana-central $75 \%$ $(9 / 12)$, havendo também moradores de chácaras em áreas periféricas $(25 \%, 3 / 12)$. Com relação à distribuição pelos bairros, houve uniformidade, ou seja, de modo geral houve apenas um caso por bairro, com exceção do bairro Jardim Capelinha, que abriga dois casos.

O perfil dos acumuladores de animais foi caracterizado por mulheres $91,67 \%$ (11/12), com idade média de 62,17 anos, solteiras $(66,66 \%)$, residindo em casa própria $(75 \%, 9 / 12)$, vivendo sozinhas $(50 \%, 6 / 12)$, em sua maioria desempregadas $(41,67 \%, 5 / 12)$ ou aposentadas $(41,67 \%, 5 / 12)$; e, ainda, $8,3 \%$ $(1 / 12)$ catadora de materiais recicláveis e $8,3 \%$ (1/12), sem registro de ocupação específica. Também foram registradas comorbidades, ou seja, doenças associadas, em alguns indivíduos. Observou-se a presença de hipertensão e diabetes (25\%, 3/12); alcoolismo (16,6\%, 2/12); depressão (16,6\%, $2 / 12)$; surdez $(8,3 \% 1 / 12)$; artrose $(8,3 \%, 1 / 12)$ e epilepsia $(8,3 \%, 1 / 12)$. Sobre antecedentes familiares e emocionais, foram registados ocorrência de incêndio residencial $(16,6 \%$, $2 / 12)$, acidente vascular cerebral(AVC) de cônjuge $(8,3 \%, 1 / 12)$, morte de cônjuge $(8,3 \% 1 / 12)$, presença de usuário de drogas na família $(8,3 \% 1 / 12)$ e filho com transtorno psiquiátrico $(8,3 \%$ $1 / 12)$. Destes, $66,67 \%(8 / 12)$ recebem acompanhamento pelas Unidades Básicas de Saúde, por meio de serviços de saúde básica e acompanhamento psicológico. Os principais animais acumulados foram cães e gatos, porém houve relatos de outras espécies como galinha, pato e jabuti (figura 02). O número médio de animais por residência foi 49,3 . Destes acumuladores de animais, 66,67\%(8/12) também acumulavam algum tipo de objetos, e lixo $91,67 \%(11 / 12)$.

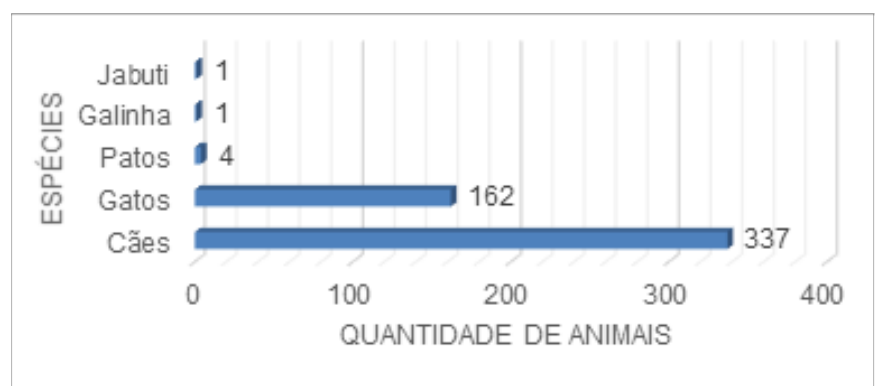

Figura 2: Animais acumulados no município de Guarulhos, segundo espécie.

Sobre as punições e ações decorrentes ao acúmulo, $58,3 \%$ (7/12) receberam multas por infrações municipais e 33,33\% (4/12) por processos judiciais, sendo destes $75 \%$ (3/4) por processos ambientais. Ao todo, $100 \%(12 / 12)$ dos casos ainda estão em andamento, ou seja, nenhum deles foi concluído ou finalizado até a conclusão deste levantamento.

$O$ instrumento de vistoria em atenção a indivíduos em situação de acúmulo de animais (figura 03) foi elaborado subdividido em cinco partes. Na parte inicial está a caracterização do indivíduo (nome, idade, endereço, contato, número do processo, comorbidades, acompanhamento psicológico e/ou adesão, familiares e origem da denúncia). Na segunda parte, seguem os dados referentes a caracterização dos animais (espécie, quantidade, estado de saúde); na terceira parte a descrição das condições gerais do ambiente (funcionalidade do ambiente, acesso, presença de lixo, animais sinantrópicos e vizinhança); na quarta estão presentes as condições dos animais (condições de saúde e nutrição e, ainda, indicadores de comprometimento crônico de bem-estar animal ou maustratos), e na quinta e última parte, estão presentes o campo "desfecho" para elucidar o seguimento do caso e o campo "outras informações" para o agente descrever informações complementares que julgar necessárias. Em todas as partes do formulário haverá itens de múltipla escolha, para facilitar e otimizar o preenchimento. 


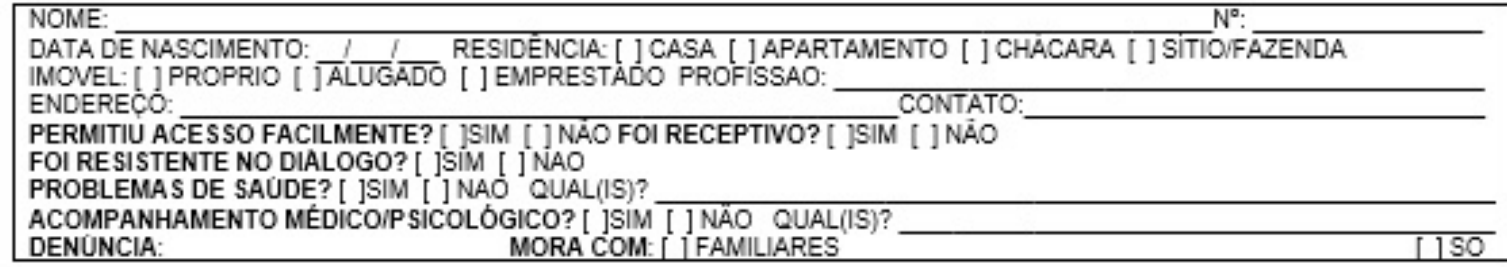

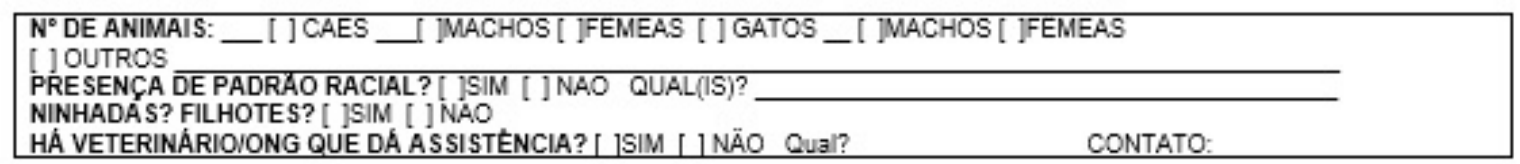

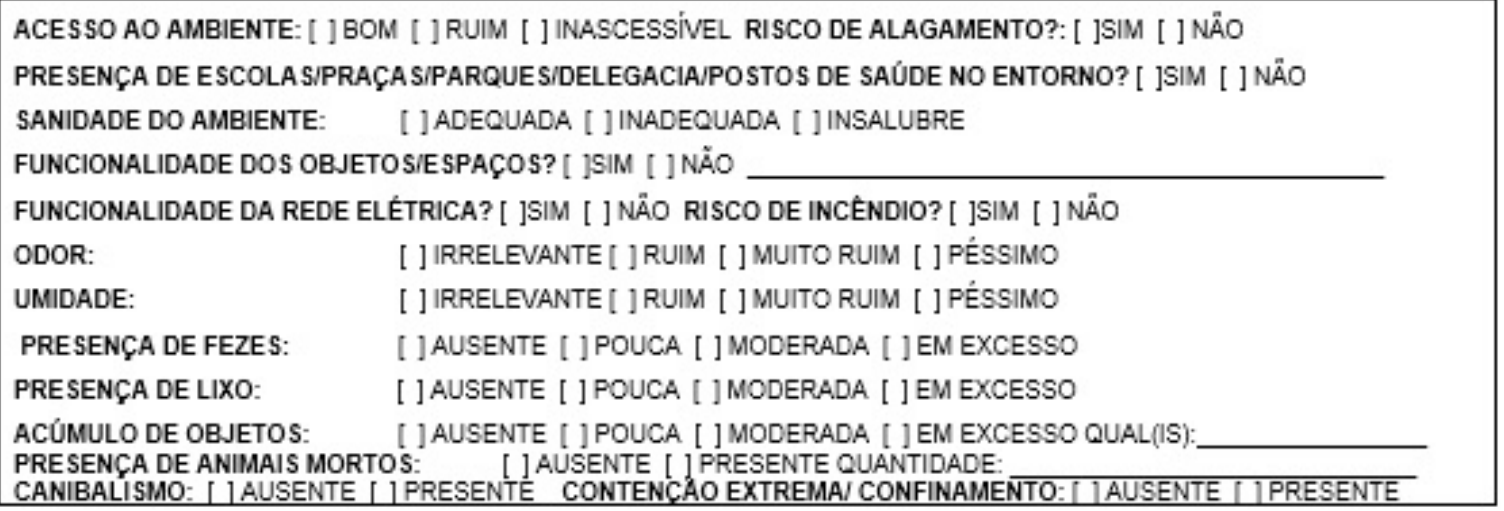

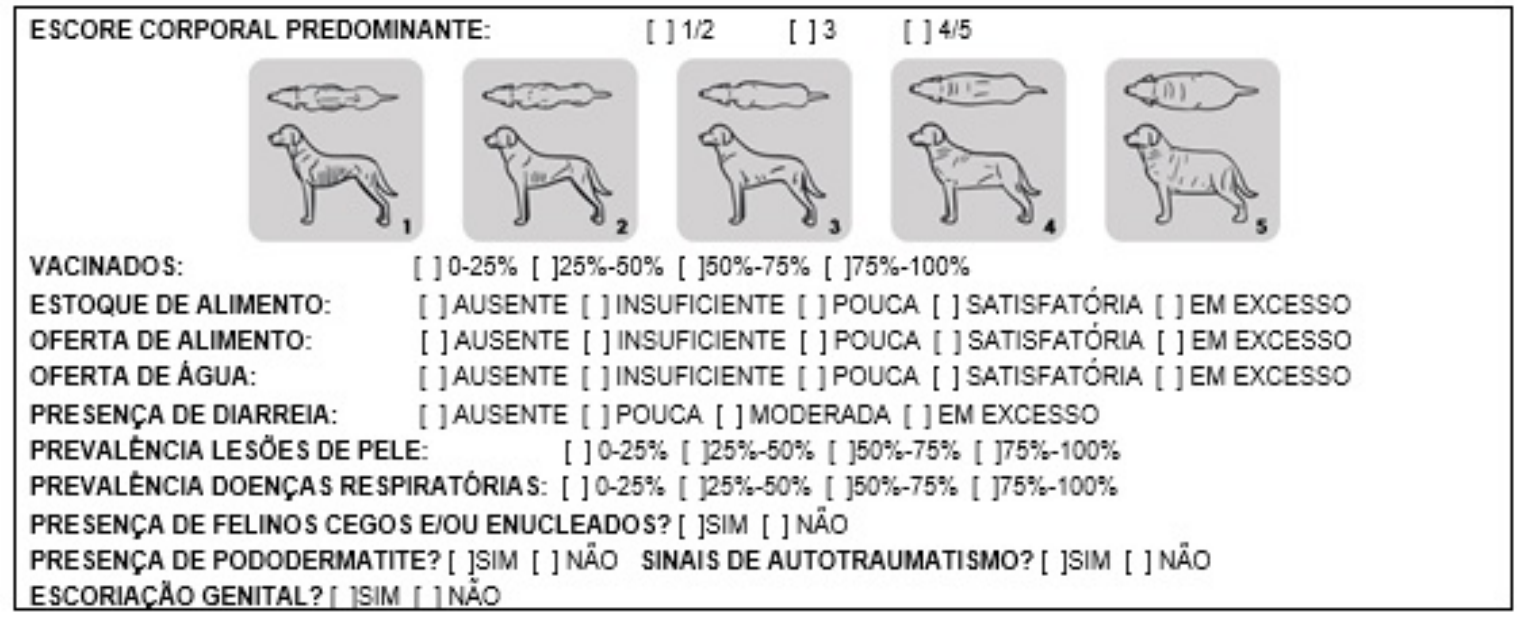

\section{OUTRAS INFORMAÇÖES: DESFECHO/ÓBITO?:}

\section{NOME (DE QUEM VISTORIOU): ASSINATURA:}

Figura 3: Modelo proposto de instrumento de vistoria técnica em casos de acumuladores de animais* ${ }^{*} \mathrm{O}$ autor disponibiliza, por e-mail, o arquivo em pdf.

\section{Discussão}

O transtorno de acumulação de animais é um fato complexo, significativo na sua frequência de ocorrência, de ampla distribuição e que deve ser discutido. Considerando os dados estudados, as consequências do transtorno repercutem negativamente na vida social do indivíduo, e possivelmente nas relações entre vizinhos, pois pelo observado no motivador das denúncias identificadas, a preocupação com os animais pelo excesso de agrupamento e condições de maus-tratos - e o incômodo por eles gerado foram os principais mobilizadores de denúncia. De acordo com os resultados obtidos, o ano de 2015 foi o responsável pelo maior número de denúncias, $41,7 \%$, possivelmente pela maior visibilidade da RIAPAC, por 
meio do reconhecimento da população e de outros agentes de saúde, decorrentes da realização de eventos multi-setoriais realizados no ano anterior, 2014. Ressalta-se que, na dinâmica deste transtorno, o número de animais e suas condições são os principais fatores, que além de ajudar a caracterizar a psicopatologia do indivíduo, são aqueles que causam maior incômodo social, principalmente aos moradores da região e vizinhos. É fundamental considerar se os moradores do entorno, ao vivenciar esta situação, se preocupam com a transmissão de zoonoses; ou seja, se a população também leva em consideração o risco iminente de contrair uma doença possivelmente transmitida pelos excessos resultantes do acúmulo de animais.

Outro fator relevante a ser comentado, é a visão distorcida da sociedade considerando o acumulador como aquele que, propositalmente, promove maus-tratos aos seus animais. Essa postura demonstra o errôneo dimensionamento do problema, sem a consideração da sua real complexidade, sem entender que o transtorno de acumulação é uma psicopatologia que deve ser corretamente atendida nas suas amplas necessidades. Campanhas informativas que expliquem à sociedade a dinâmica deste fato, e suas interferências na vida social do acumulador; em suas relações familiares e em sua saúde, deixando claro de que se trata de uma doença que acomete o indivíduo fazendo com que não haja percepção das condições de seus animais, podem facilitar o processo de compreensão deste quadro. Com um melhor entendimento social, a população poderá atuar auxiliando os serviços de saúde por meio do acompanhamento do caso, e possivelmente no sentido de estabelecer vínculo com o indivíduo acumulador, auxiliando no processo de adesão dele ao tratamento psiquiátrico, por exemplo.

Quando se realiza uma denúncia, o denunciante tem a expectativa de que os órgãos competentes intervenham e solucionem o problema. Atualmente, sabe-se que é necessária abordagem mais ampla. O trabalho inovador da RIAPAC, como uma rede intersetorial vem sendo de extrema importância para o correto acompanhamento destes casos no município de Guarulhos; pois, através da atuação "em rede", o acumulador é atendido nas suas amplas necessidades, através do encaminhamento para o acompanhamento médico-psicológico, por exemplo. Com relação à casuística e o perfil de acumuladores de animais, no município estudado identificou-se que a idade média foi de 62,2 anos. Devemos levar em consideração que nesta faixa etária, os excessos do acúmulo começam a ser mais facilmente percebidos pela vizinhança, pois, devido à idade, as consequências acabam sendo mais evidentes devido à vulnerabilidade presente na fase idosa, além disso, indivíduos idosos em situações de risco acabam causando maior comoção social, consequentemente aumentando as denúncias.

Considerando a literatura consultada, a variação identificada em casos de acumuladores em diferentes municípios, estados, ou até mesmo países envolvidos, se dá, geralmente, devido à conduta adotada por parte de órgãos públicos frente aos animais. No município de Guarulhos, as medidas dispensadas, especificamente, aos animais variam de acordo com o caso, pois a legislação vigente não permite a remoção deles pelo simples fato de serem mantidos por acumuladores e ainda não haveria local para destinação e tratamento dos animais removidos. Em alguns casos eles são retirados apenas após ação judicial. De modo geral, no município de Guarulhos, são oferecidas castrações aos cães e gatos, visando o controle populacional, e a vacinação antirrábica.

Os animais mais acumulados seguiram a tendência dos casos internacionais, portanto, cães e gatos - especialmente cães - estiveram mais comumente presentes no cenário deste transtorno. Vale ressaltar que, cães são mais facilmente percebidos pelos vizinhos e sociedade, devido ao barulho gerado; desta forma, o menor número registrado de casos de acumuladores de gatos pode estar relacionado ao fato deles serem menos denunciados devido à ausência de ruídos, causando menor incomodo aos moradores próximos em comparação aos cães.

No serviço de vigilância em saúde de Guarulhos, atualmente, a coleta de dados, durante uma inspeção técnica nos casos de acúmulo de animais, não é padronizada; portanto, as informações contidas em um relatório de vistoria sofrem variações de acordo com o profissional que as coletou, bem como, a interpretação pessoal dele, havendo interferência no registro da situação vistoriada. Da forma como vem acontecendo, muitas informações podem não ser registradas, ocorrendo escassez de dados importantes que poderiam contribuir para melhor entendimento do caso e, consequentemente, atendimento e resolução dele. Portanto, um agente de saúde no momento da vistoria, por exemplo, pode se atentar mais a quantidade dos animais do que nas condições física ou comportamental deles, ou determinada condição do animal chame mais atenção a ele do que a um outro profissional em uma próxima vistoria, e vice-versa. Os agentes são profissionais de diferentes áreas de formação acadêmica, consequentemente, tende-se um olhar para as áreas com maior aderência da sua formação acadêmica, é natural, mas, isso não é bom no atendimento de uma situação complexa.

As pesquisas sobre o transtorno de acumulação de animais são recentes e escassas, não havendo, ainda, uma uniformidade nas ações em atendimento ao caso. O que se pode afirmar é que há mobilização de muito sofrimento, o acumulador e familiares sofrem, os animais e a população do entorno sofrem, a sociedade que refuta a situação, sofre. $O$ atendimento dos casos de acúmulo de animais em Guarulhos, na sua maioria, está sob a atenção da RIAPAC há mais de quatro anos, e sem previsão de resolução, demonstrando assim, a complexidade do tema.

\section{Conclusões}

O perfil de acumuladores de animais no município de Guarulhos se caracteriza por mulher, solteira, idosa desempregada ou aposentada, residente sozinha em casa própria. Há também a presença de fatores psicológicos associados ou comorbidades, acumulando cães e gatos, especificamente, cães em sua maioria. Com base no levantamento, um instrumento de coleta de dados foi elaborado para contribuir na fase de captação das informações, padronizando os dados tão importantes para o entendimento da situação para correta intervenção. Diante do exposto, tal transtorno apresenta elevada relevância social, porém, apesar de muito presente, nossa sociedade e serviços públicos ainda precisam compreendê-lo de maneira global para corretas intervenções. Desta forma, justifica-se a importância de mais estudos de abrangência nacional que contribuirão com os serviços municipais melhorando o bem-estar dos indivíduos, por meio de corretas abordagens e adesão aos tratamentos, e dos animais em situação de acúmulo. 


\section{Referências}

APA - American Psychiatric Association. Diagnostic and Statistical Manual of Mental Disorders. 5. ed. Arlington, VA: American Psychiatric Association, 991 p. 2013a.

APA - American Psychiatric Association. Hoarding Disorder. Disponível em: http://www.psychiatry.org/mental-health/key-topics/ hoarding-disorder Acesso em 23 mai 2016. 2013b.

APA - American Psychiatric Association. Manual Diagnóstico e Estatístico de Transtornos Mentais (DSM-5). São Paulo, SP: Artmed. 2014.

BARBOSA E.F. Instrumento de coleta de dados em pesquisas educacionais. 05/12/2008..Disponível.em:.<http://www.inf.ufsc. br/ vera.carmo/Ensino_2013_2/Instrumento_Coleta_Dados Pesquisas_Educacionais.pdf>. Acesso em: 06 dez. 2017, 2008.

CUNHA G.R., MARTINS C.M., CECCON-VALENTE M.F., SILVA L.L., FLOETER, D., ROBERTSON J.V., FERREIRA, A.W., BIONDO A.W. Frequency and spatial distribuition of animal and object hoarder behaviour in Curitiba, Paraná State, Brazil. Cadernos de Saúde pública. 2017, vol. 33, n.2, e1316.

DIMAURO J., TOLIN D.F., FROST R.O., STEKETEE G. Do people with hoarding disorder under-report their symptoms?. Journal of Obsessive-Compulsive and Related Disorders. 2(2), 130-6. 2013.

DOMINGUES L.R., CESAR J.A., FASSA A.G., DOMINGUES M.R. Guarda responsável de animais de estimação na área urbana do município de Pelotas, RS.. ciência e saúde coletiva 2013/Out). Disponível em: <http://www.cienciaesaudecoletiva. com.br/artigos/guarda-responsavel-de-animais-de-estimacao-naarea-urbana-do-municipio-de-pelotas-rs/14528> Acesso em 03 jun 2018, 2013.

GUARULHOS, SP. Lei ${ }^{\circ}$ 6.144, de 7 de junho de 2016,

GUARULHOS, SP. Portaria 158/2018-SS, de 21 de setembro de 2018.

GUARULHOS, Departamento de Proteção Animal de Guarulhos. Disponível em: <http://dpan.guarulhos.sp.gov.br/ > Acesso em 03 jun 2018, 2018.

GRISHAM J.R., Norberg M.M. Compulsive Hoarding: current controversies and new directions. Dialogues in Clinical Neuroscience, 12(2), 233-40. 2010.

GRISHAM J.R., FROST R.O., STEKETEE G., Kim H.J., Hood $\mathrm{S}$. Age of onset of compulsive hoarding. Journal of Anxiety Disorders, 20(5), 675-86. 2016.

HARC- Hoarding of Animals Research Consortium. Disponível em: <https://vet.tufts.edu/hoarding/> Acesso em 28 mai 2106, 2016.

IBGE, Instituto Brasileiro de Geografia e Estatísticas. População de animais de estimação no Brasil - 2013. Disponível.em:.<http:// www.agricultura.gov.br/arq_editor/file/camaras_tematicas/ Insumos_agropecuarios/79RO/IBGE_PAEB.pdf > Acesso em 28 mai 2016, 2013.

IBGE, Instituto Brasileiro de Geografia e Estatísticas. Município de Guarulhos. Disponível em: <https://cidades.ibge.gov.br/brasil/ sp/guarulhos>. Acesso em 03 jun 2018, 2018.

LIMA R. Acumuladores compulsivos - uma nova patologia psíquica. Revista Espaço Acadêmico, n 126 , novembro 2011, ano XI, isnn 1519-6186. http://www.periodicos.uem.br/ojs/index. php/EspacoAcademico/article/view/15203/8158. Acesso em 28 mai 2016, 2011.
MATAIX-COLS D., PERTUSA, A. Annual research review: Hoarding Disorder: potential benefits and pitfalls of a new mental disorder. Journal of Child Psychology and Psychiatry, 53(5), 60818. 2012.

MILLS, $\mathrm{H}$. The role of trauma in hoarding disorder. (Dissertação de Mestrado). Disponível em: <https://dspace.smith.edu/ handle/11020/24225> Acesso em: 05 jun 2017, 2013.

PATRONEK G.J., NATHANSON J.N. ANIMAL HOARDING: Structuring Interdisciplinary Responses to Help People, Animals, and Communities at Risk. Hoarding of Animals Research Consortium. 2006.

PATRONEK, G.J. Hoarding of Animals: an under-recognized public health problem in a difficult - study population. Public Health Report. Jan/Fev 1999. Volume 114. 1999.

PATRONEK G.J., NATHANSON J.N. A theoretical perspective to inform assessment and treatment strategies for animal hoarders. Clinical Psychology Review, 29(3), 274-81, 2009.

PERTUSA A., FULLANA M.A., SINGH S., ALONSO P., MENCHÓN J.M., MATAIX-COLS D. Compulsive Hoarding: OCD symptom, distinct clinical syndrome, or both?. American Journal of Psychiatry, 165(10), 1289-98. 2008.

PERTUSA A., FROST R.O., FULLANA M.A., SAMUELS J., STEKETEE G., TOLIN D., SAXENA S., LECKMA J.F., MATAIXCOLS D. Refining the diagnostic boundaries of Compulsive Hoarding: a critical review. Clinical Psychology Review, 30(4), 371-86. 2010.

POGOSIAN, L. Treatment of compulsive hoarding: a case study. The Einstein Journal of Biology and Medicine, 25(1), 8-11. 2010.

ROCHA S.M., CUNHA G.R., BIONDO A.W. Perfil das denúncias de acumuladores de animais da cidade de Curitiba-Pr. Revista de Educação Continuada em Medicina Veterinária e Zootecnia, v. 13, n. 2. 2015.

SÃO PAULO, SP. Lei no 13.725, de 09 de janeiro de 2004. SÃO PAULO, SP. Decreto $n^{\circ} 57.5270$, de 28 de dezembro de 2006.

SNOWDON J., PERTUSA A., MATAIX-COLS D. ON HOARDING AND SQUALOR: A FEW CONSIDERATIONS FOR DSM5, Depression and Anxiety, 29, 5, (417-424), 2012.

STEKETEE G., FROST R. Compulsive Hoarding: current status of the research. Clinical Psychology Review, 23(7), 905-27. 2003.

TAVOLARO P., CORTEZ T.L. A Acumulação de animais e a formação de veterinários. Atas de Saúde Ambiental (São Paulo), ISSN: 2357-7614 - Vol. 4, JAN-DEZ, 2016, p. 161-178. 2016.

TOLIN D.F., FROST R.O., STEKETEE G., GRAY K.D., FITCH K.E. The economic and social burden of compulsive hoarding. Psychiatry Research, 160(2), 200-11. 2008.

VALENTE S.M. The Hoarding Syndrome: screening and treatment. Home Healthcare Nurse, 27(7), 432-40. 2009.

WHO- World Health Organization. One Health. September 2017. Disponível em: <http://www.who.int/features/qa/one-health/en/>. Acesso em: 05 dez. 2017, 2017. 